\title{
Daily consumption of a synbiotic yogurt decreases energy intake but does not improve gastrointestinal transit time: a double-blind, randomized, crossover study in healthy adults
}

Hilary M F Tulk ${ }^{\dagger}$, Diane C Blonski ${ }^{\dagger}$, Lauren A Murch, Alison M Duncan and Amanda J Wright ${ }^{*}$

\begin{abstract}
Objective: Probiotic and synbiotic products are widely marketed to healthy individuals, although potential benefits for these individuals are rarely studied. This study investigated the effect of daily consumption of a synbiotic yogurt on gastrointestinal (Gl) function in a sample of healthy adults.

Subjects/Methods: In a randomized crossover double-blind study, 65 healthy adults consumed $200 \mathrm{~g} /$ day of yogurt with (synbiotic) or without (control) added probiotics (Bifidobacterium lactis Bb12, Lactobacillus acidophilus La5, Lactobacillus casei CRL431) and $4 \mathrm{~g}$ inulin for two 15-day treatment periods, each preceded by a 6-week washout period. Gl transit time (GTT), duration of colour (DOC), Gl symptoms and dietary intake were assessed and analyzed using repeated measures ANOVA, including PRE-treatment GT as a covariate. Participants were grouped as short GTT (ST, $n=50, \leq 32.7$ h) or long GTT (LT, $n=15,>32.7$ h) based on their PRE-treatment GT assessment.

Results: POST-treatment GT and DOC were not different between synbiotic and control, and did not change from PRE-treatment, within the STT or LTT groups. There were no changes in Gl symptom ratings, indicating that both yogurts were well tolerated. In STT, energy, fat and protein intakes were decreased from baseline with synbiotic ( $p=0.055, p=0.059$ and $p=0.005$, respectively) and dietary fibre intake was higher POST-treatment with synbiotic versus control $(p=0.0002)$. In LTT, decreases in energy and fat intakes with synbiotic were not significant $(p=0.14$ and $p=0.18$, respectively) and there were no differences in dietary fibre intake.
\end{abstract}

Conclusion: Consuming $200 \mathrm{~g} /$ day of synbiotic yogurt did not significantly alter GTT in healthy adults, but was well tolerated and helped to reduce overall energy intake.

Keywords: Synbiotics, Yogurt, Gastrointestinal transit, Energy intake

\section{Introduction}

It is well established that the gastrointestinal (GI) microbiota impacts overall health and that the quantities and types of bacteria composing the microbiota can be optimized by dietary factors, particularly prebiotics and probiotics [1]. Consequently, consumers are turning to probiotic-containing foods for relief of specific conditions or to improve overall health [2]. However, evidence to support health claims for probiotics and synbiotics

\footnotetext{
*Correspondence: ajwright@uoguelph.ca

${ }^{\dagger}$ Equal contributors

Department of Human Health and Nutritional Sciences, University of Guelph, Guelph, Ontario N1G 2W1, Canada
}

\section{() BioMed Central}

(containing prebiotics and probiotics) in the general population, the main group to whom these products are marketed, is limited [2,3]. The efficacy of synbiotics can vary depending on the probiotic strain and the food matrix can affect probiotic survival and potential health benefits $[4,5]$. Strain- and product-specific research to substantiate the efficacy of probiotics in improving or maintaining GI health in healthy consumers is required [2-4,6].

Probiotics have traditionally been used to treat GI diseases and disturbances, including lactose intolerance, rotavirus and antibiotic associated diarrhea, inflammatory bowel disease and constipation [3,7,8]. Consumption of fermented dairy products containing Bifidobacterium lactis 
DN-173010 ( $\left.\geq 10^{8} \mathrm{CFU} / \mathrm{g}\right)$ has been shown to improve bowel movement frequency and consistency in women with constipation [9], to decrease initially long GI transit times (GTT) in healthy women [10], men [11] and older adults $[12,13]$, and to improve overall GI well-being and digestive symptoms in healthy women [14]. Intestinal transit time tended to decrease $(\mathrm{p}=0.055)$ in healthy Japanese women with slow transit times ( $>40$ hours) whom consumed $170 \mathrm{~g} /$ day yogurt containing $10^{8} \mathrm{CFU} / \mathrm{g}$ of the same probiotic strain [15].

A growing body of work exists with respect to potential GI health benefits of dairy products containing various probiotic strains [16-18] including Bifidobacterium lactis Bb12 and Lactobacillus acidophilus La5 [19-21]. Savard et al. [2011] showed that these probiotics consumed for 4 weeks from a commercial yogurt survived GI transit and led to increases and decreases, respectively, in the presence of beneficial and potentially pathogenic gut bacteria. Consumption of yogurts containing $\mathrm{Bb} 12$, in combination with Lactobacillus acidophilus La5, has also been shown to suppress $\mathrm{H}$. pylori infections [22], to improve antioxidant status in participants with type 2 diabetes [23], and to maintain serum insulin levels in pregnant women [24].

Prebiotics are non-digestible carbohydrates that selectively stimulate the growth of beneficial bacteria in the GI tract [25]. Fructooligosaccharides, in particular inulin, are commonly added to foods in concentrations from 0.6-2.1 g/100 g [1]. Inulin is a soluble prebiotic fibre that has been shown to increase fecal Bifidobacteria spp. levels [26], to increase stool bulk and frequency when initially low [27], and to exert a mild laxative effect in elderly people with constipation [28]. As well, $16 \mathrm{~g} /$ day of inulin and oligosaccharides decreased subjective hunger scores and increased concentrations of plasma gut peptides that regulate food intake, suggesting a satiety effect in healthy adults [29]. However, research to establish the relevance of satiety-promoting foods, in terms of their long-term ability to modulate food intake and to beneficially impact body weight, is required. Also, while there is rationale for enriching foods with prebiotics, some studies have associated prebiotic consumption, including inulin, with mild GI side effects [29-31]. Since functional foods that modulate energy and increase fibre intake may be an important strategy in addressing overweight, obesity and their associated conditions [29,31,32], research addressing the tolerance of prebiotics in the general population is warranted.

Consumption of dairy products, including yogurt, by North American and European consumers is considerable and dairy products currently lead the probiotic market [33]. This is, in part, due to the healthy perceptions and familiarity consumers have with fermented dairy products containing live bacteria [5] and to the fact that live strains of probiotics can be added to these products relatively easily [34]. Yogurt, in particular, is considered to be a suitable synbiotic food matrix [34] since it is intrinsically thickened and its texture should remain acceptable if prebiotic fibre-addition induces further thickening [35]. Addition of a prebiotic to dairy products containing probiotics has also been shown to improve the survival of probiotic bacteria during their shelf-life [36,37]. Therefore, synbiotics are an exciting area of growth for the dairy sector [1]. More broadly, a growing body of evidence supports a role for various synbiotic supplements and foods in improving GI disturbances [14,38-42].

There is also evidence that fibre-enriched yogurts can suppress short-term appetite and may be effective in controlling food intake [43,44]. For example, yogurt was found to have greater satiating effects than isocaloric fruit-based or dairy fruit beverages [45] and the effect was enhanced when yogurt was enriched with $6 \mathrm{~g}$ of inulin [46]. Therefore, optimizing the microbial population of the GI tract through the consumption of synbiotics could be a strategy in preventing obesity, in addition to other chronic diseases such as inflammatory bowel disorders, and some cancers $[3,29,32,47]$. This study investigated whether daily consumption of a synbiotic yogurt improves GI function in a sample of healthy Canadian adults. GTT, GI symptoms and food intake were compared between participants with short or long baseline GTT following consumption of a synbiotic yogurt or a control product without added probiotics and inulin.

\section{Methods}

\section{Study design}

This study was conducted at the Human Nutraceutical Research Unit (HNRU) in the Department of Human Health and Nutritional Sciences at the University of Guelph and was approved by the University's Human Research Ethics Board. A randomized crossover double-blind design was utilized and consisted of two 15-day treatment periods, each preceded by a 6 -week washout period.

\section{Participants}

Healthy males and females (18 to $65 \mathrm{y}$, BMI $18-35 \mathrm{~kg} / \mathrm{m}^{2}$ ) were recruited from the local community and pre-screened using a phone/email questionnaire. Individuals were excluded if they had food allergies or intolerances, GI disorders or disease or if they regularly used medications (except hormonal contraceptives), natural health products or dietary supplements, or had used antibiotic medication $<3$ months prior to the study. Individuals who smoked or were elite or varsity athletes and/or training for a major athletic event, and females who were pregnant, lactating or not practicing birth control, were also excluded. All participants provided written informed consent and attended an orientation session prior to the study. 


\section{Determination of gastrointestinal transit time}

GTT was measured using capsules containing $0.25 \mathrm{~g}$ of carmine red (Sensient Colours Canada Ltd., Kingston, ON) and $0.15 \mathrm{~g}$ of carbon black (Castleguard Health Services, Paris, ON). Carmine red is a non-digestible food colourant that produces a distinct red colour in the feces $[48,49]$ which is enhanced by the presence of carbon black [13].

Participants consumed $2.0 \mathrm{~g}$ carmine red and $0.5 \mathrm{~g}$ carbon black with breakfast on two mornings, separated by $72 \mathrm{~h}$, for duplicate determination of GTT. They recorded the date and time of capsule consumption and the date, time and colour of all subsequent bowel movements until both doses of colour had passed. GTT was defined as the length of time (h) between capsule consumption and appearance of red colour in the feces and is reported as the average time for both doses to appear in the feces. The duration of red colour (DOC) in the feces was also calculated, as a secondary indicator of GI transit. DOC was defined as the length of time (h) between capsule consumption and the last appearance of red colour in the feces and is reported as the average time for both doses to disappear from the feces. DOC relates to the amount of time the bowel mucosa is exposed to digesta, including possible carcinogens [49].

At the start of the 6-week washout period, a baseline GTT assessment was completed to familiarize participants with the GTT assessment protocol and to screen for participants with difficulty detecting the red colour in their feces. Baseline GTT data was used for preliminary examination of the distribution of GTT within participants. GTT assessments were completed PRE-treatment (commencing 3 days prior to Day 1) and POST-treatment (commencing on Day 13) during each 15-day treatment period.

\section{Study treatments}

Participants were instructed to maintain their usual lifestyle and dietary habits with specific instructions to avoid yogurt products and foods with added prebiotics or probiotics, with the exception of the study treatments. The study treatments were industrially manufactured vanilla flavoured yogurts with (symbiotic) and without (control) the addition of Bifidobacterium lactis Bb12 ( $\left.\geq 10^{7} \mathrm{CFU} / \mathrm{g}\right)$, Lactobacillus acidophilus La5 ( $\left.\geq 10^{7} \mathrm{CFU} / \mathrm{g}\right)$, Lactobacillus casei CRL431 ( $\left.\geq 10^{7} \mathrm{CFU} / \mathrm{g}\right)$ and $2 \mathrm{~g}$ inulin (average degree of polymerization $\geq 10$, Beneo Raftiline) per $100 \mathrm{~g}$ serving. Probiotic counts $\geq 10^{9} \mathrm{CFU}$ per $100 \mathrm{~g}$ serving were verified by pour plate count methods throughout the treatment period. Both yogurts were produced using identical starter cultures and milk ingredients and were manufactured for each treatment period separately so that the products were consumed at the same point in their shelf life. Treatments were packaged as $100 \mathrm{~g}$ servings in opaque white cups, stored under refrigeration and provided to participants immediately prior to the start of each treatment period. Per $100 \mathrm{~g}$ serving, the yogurts contained $100 \mathrm{kcal}, 3.0$ g protein, 18.0 g carbohydrate and $3.0 \mathrm{~g}$ fat. Participants were instructed to consume two $100 \mathrm{~g}$ servings of yogurt per day during each treatment period and to keep the yogurt refrigerated. Participants recorded the date, time, and other details about their yogurt consumption in a daily study diary.

\section{Baseline anthropometric data}

Five days prior to the first treatment period, participants reported to the HNRU after a $12-14 \mathrm{~h}$ overnight fast. Height was measured to the nearest $0.1 \mathrm{~cm}$ using a stadiometer (SECA Portable Stadiometer 214, Hanover, MD, USA) and body weight was measured to the nearest $0.1 \mathrm{~kg}$ using a calibrated digital scale (Acculab SV-100, Edgewood, NY, USA). Body fat (\%) was determined by bioelectric impedance analysis (BodyStat $1500^{\mathrm{m}}$, BodyStat Ltd., Douglas, IOM).

\section{Gastrointestinal symptoms}

A modified version of the GI symptom rating scale (GSRS) [50] was used to evaluate changes in perceived GI symptoms at Days 1, 7 and 15 of each treatment period. The modified GSRS included 5 questions pertaining to abdominal discomfort, stomach grumbling, bloating, belching, and flatulence, each scored as 0 for 'no symptoms' to 3 for 'extreme symptoms', and one question each related to consistency and frequency of bowel movements scored as 0 to 4 , where 0 and 4 indicated opposite extremes and 2 indicated normal consistency or frequency.

\section{Dietary intakes}

Three-day food records were collected at the start of the first 6-week washout period (baseline) and on Days 13-15 of each treatment period (POST-treatment). Participants were provided with detailed instructions for food record completion. Food records were reviewed with a study coordinator upon submission, analyzed using The Food Processor ${ }^{\oplus}$ SQL version 10.3.0.0 (ESHA Research Salem, OR, USA) and 3-day means for energy, fat, protein, carbohydrate and dietary fibre were calculated.

\section{Data and statistical analysis}

The intra-individual variation in PRE-treatment GTT assessment data was examined for each participant. Participant data was excluded from statistical analysis if the PRE-treatment GTT was significantly different between synbiotic and control or if the coefficient of variation for PRE-treatment GTT was $\geq 40 \%$. Participants were grouped as short GTT (STT) or long GTT (LTT) based on [13] and [12]. The distribution of PRE-treatment GTT was 
examined using a histogram to determine a cut-off point for grouping of participants as STT or LTT (Figure 1).

GTT was analyzed for effect of treatment within STT and LTT groups, both between treatments (using repeated measures ANOVA on POST-treatment values and controlling for participant, treatment and treatment order and including PRE-treatment values as a covariate), and within treatments (using a paired $t$-test on PRE- and POST-treatment values within a treatment). Baseline anthropometric data were compared between STT and LTT groups using an unpaired $t$-test. GSRS scores were analyzed within STT and LTT groups for effect of time (i.e. Days 1 vs. 7 vs. 15) within treatments and for the effect of treatment within study days, using Chi squared analysis. Dietary intake data was analyzed within STT and LTT groups for effect of time (i.e. baseline vs. POST-treatment) within treatments and for effect of treatment (on POST-treatment values), both using unpaired t-tests. All statistical analyses were performed using SAS version 9.1 (SAS Institute, Inc., Cary, NC) and a significance level of $\mathrm{p}<0.05$.

\section{Results}

\section{Participant flow and GTT grouping}

Of the 300 potential participants screened by phone or email, 158 attended a screening appointment and 127 were eligible for the study, assigned participant numbers and invited to complete a baseline GTT assessment. 111 participants completed the baseline GTT assessment. Of these, 28 subsequently dropped out or were excluded from the study due to scheduling conflicts $(n=9)$, use of antibiotics or other medications $(n=3)$, difficulty swallowing the capsules $(n=2)$, GI upset or diarrhea $(n=6)$, or not wishing to continue $(n=8)$. Therefore, 83 participants entered the first treatment period, during which 4 participants discontinued their participation due to illness $(n=2)$, scheduling conflicts $(n=1)$, and antibiotic use $(n=1)$. After completion of the first treatment period, 10 participants were excluded from the study due to GI upset (i.e. diarrhea) during the GTT assessments $(n=5)$, non-compliance to study protocol $(n=1)$, commencement of antibiotics or prescription drugs $(n=2)$ or scheduling conflicts $(n=2)$. After analysis of the PRE-treatment GTT for intraindividual variation $\geq 40 \%, 4$ more participants were excluded. The distribution of PRE-treatment GTT for each treatment period was examined for the 65 participants whose data was included in the statistical analysis. GTT data was divided at the 75 th percentile with participants grouped as either short GTT (STT; $\mathrm{n}=50, \leq 32.7 \mathrm{~h}$ ) or long GTT (LTT; $\mathrm{n}=15,>32.7 \mathrm{~h}$ ). Sample size was estimated at 41 participants for the STT group and 17 participants for the LTT group using effect sizes (i.e. time reductions) of 4 hours for STT and 10 hours for LTT, standard deviation estimates of 5 for STT and 8 for LTT, a power of 95\%, an alpha of 0.05 and 2-sided testing.

\section{Participant characteristics}

At baseline, participants had a mean age of $28.6 \mathrm{y}$, body weight of $72.1 \mathrm{~kg}$, height of $171.2 \mathrm{~cm}, \mathrm{BMI}$ of $24.4 \mathrm{~kg} / \mathrm{m}^{2}$ and body fat of $23.0 \%$. There were no significant differences in participant characteristics between the STT and LTT groups (Table 1). The STT group consisted of 23 men and 27 women, whereas the LTT group consisted predominantly of women $(n=15)$ versus men $(n=3)$.

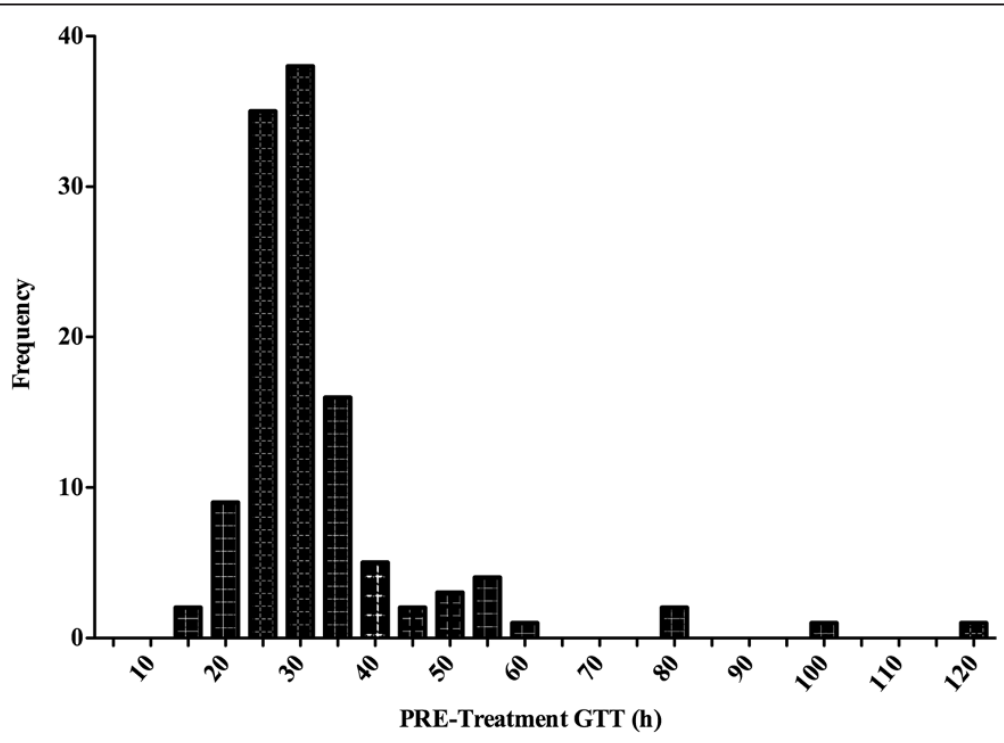

Figure 1 Histogram depicting the distribution of PRE-treatment GTT ${ }^{1}$. Abbreviations: GTT, gastrointestinal transit time. ${ }^{1}$ Distribution of PRE-treatment GTT values, measured in duplicate in two separate GTT assessments, for each participant $(n=65)$. 
Table 1 Baseline characteristics for all participants and for STT and LTT groups ${ }^{1}$

\begin{tabular}{lccc}
\hline & $\begin{array}{c}\text { All participants } \\
(\mathbf{n}=\mathbf{6 5})\end{array}$ & $\begin{array}{c}\text { STT } \\
(\mathbf{n}=\mathbf{5 0})\end{array}$ & $\begin{array}{c}\text { LTT } \\
(\mathbf{n}=\mathbf{1 5})\end{array}$ \\
\hline Males & $\mathrm{n}=26$ & $\mathrm{n}=23$ & $\mathrm{n}=3$ \\
Females & $\mathrm{n}=39$ & $\mathrm{n}=27$ & $\mathrm{n}=12$ \\
Age $(\mathrm{y})$ & $28.6 \pm 11.3$ & $28.0 \pm 10.6$ & $30.7 \pm 13.2$ \\
Height $(\mathrm{cm})$ & $171.2 \pm 8.9$ & $171.0 \pm 8.5$ & $171.9 \pm 8.9$ \\
Body weight $(\mathrm{kg})$ & $72.1 \pm 15.3$ & $72.4 \pm 14.1$ & $71.0 \pm 18.5$ \\
Body mass index $\left(\mathrm{kg} / \mathrm{m}^{2}\right)$ & $24.4 \pm 4.0$ & $24.6 \pm 3.5$ & $23.8 \pm 4.6$ \\
Body fat (\%) & $23.0 \pm 8.9$ & $22.4 \pm 8.5$ & $25.9 \pm 9.3$ \\
\hline
\end{tabular}

${ }^{1}$ Mean \pm SD.

\section{Gastrointestinal transit time assessments}

There were no significant differences in POST-treatment GTT or DOC between the synbiotic and control yogurts within either the STT or the LTT groups. Similarly, within the STT and LTT groups there were no significant differences between PRE-treatment and POST-treatment GTT or DOC, within either the synbiotic or control yogurts (Table 2).

\section{Gastrointestinal symptoms}

Both the synbiotic and control yogurts were well tolerated, based on participant ratings of abdominal discomfort, abdominal rumbling, abdominal bloating, belching, flatulence, bowel movement consistency and bowel movement frequency assessed by GSRS scores, within or between treatments, within both the STT or LTT groups (Figure 2).

\section{Dietary intake}

Dietary intake data is presented in Table 3. In the STT group, energy, fat and protein intakes decreased from baseline following consumption of the synbiotic yogurt ( $\mathrm{p}=0.055, \mathrm{p}=0.059$ and $\mathrm{p}=0.005$, respectively) and carbohydrate intake decreased from baseline following consumption of the control yogurt $(\mathrm{p}=0.008)$. In the LTT group, energy and fat decreased from baseline following consumption of the synbiotic yogurt, but these changes did not reach statistical significance $(\mathrm{p}=0.14$ and $\mathrm{p}=0.18$, respectively). In the STT group, dietary fibre intake did not significantly change with consumption of the synbiotic, but decreased from baseline with consumption of the control $(\mathrm{p}=0.0002)$ and was significantly higher POST-treatment with the synbiotic versus control treatment $(p=0.0002)$. In the LTT group, there was no significant change in dietary fibre intake from baseline with the synbiotic $(\mathrm{p}=0.25)$ or control $(p=0.24)$ or POST-treatment differences between the synbiotic and control $(\mathrm{p}=0.58)$.

\section{Discussion}

Although claims of improved GI function are widely implied in the marketing of probiotic and synbiotic dairy products, the roles of these products in improving GI function in healthy individuals requires further investigation. Furthermore, there are few reports of typical GTT in healthy Canadians. To our knowledge, this study is the first to examine the effects of a synbiotic yogurt on measures of GI function and dietary intake in healthy Canadian adults.

The average PRE-treatment GTT in this study was in the range of $30 \mathrm{~h}$, based on all participants. This is slightly lower than the $41.0 \pm 18.9 \mathrm{~h}$ reported for a sample of healthy Canadian adults consuming their habitual diet and determined using fecal $\mathrm{x}$-ray monitoring for passage of radiopaque pellets [51]. Consuming a synbiotic yogurt did not change GTT or DOC from baseline and was not different from the control yogurt, for either the STT or LTT groups. Past research demonstrates that DOC can vary even among individuals with the same frequency of bowel movements, particularly in those with longer transit times [49]. In this study, high variability in DOC was observed in both STT and LTT PRE- and POSTtreatment.

Our STT and LTT groups had baseline GTT $25.2 \pm$ $0.4 \mathrm{~h}$ and $49.5 \pm 4.3 \mathrm{~h}$, respectively. In a recent study, GTT, measured using the dye marker technique, was lowered in women, especially those with functional constipation (transit time $\geq 48 \mathrm{~h}$ ), whom consumed two $125 \mathrm{~g}$ servings of synbiotic yogurt containing $0.625 \mathrm{~g}$ inulin and oligofructose with Bifidobacterium lactis Bb12 $\left(10^{9}-10^{10} \mathrm{CFU} / \mathrm{g}\right)$ and Lactobacillus casei CRL

Table 2 Average GTT (h) and DOC (h) for participants with STT and LTT PRE- and POST-treatment ${ }^{1}$

\begin{tabular}{|c|c|c|c|c|c|c|}
\hline & \multicolumn{2}{|c|}{ All participants } & \multicolumn{2}{|c|}{ STT $(n=50)$} & \multicolumn{2}{|c|}{ LTT $(n=15)$} \\
\hline & Synbiotic & Control & Synbiotic & Control & Synbiotic & Control \\
\hline GTT PRE & $31.0 \pm 16.6$ & $29.8 \pm 12.7$ & $26.1 \pm 4.1$ & $24.4 \pm 4.4$ & $51.7 \pm 28.7$ & $47.8 \pm 14.8$ \\
\hline GTT POST & $30.6 \pm 18.3$ & $31.1 \pm 15.4$ & $26.3 \pm 8.2$ & $26.7 \pm 10.4$ & $48.8 \pm 33.9$ & $45.7 \pm 20.3$ \\
\hline DOC PRE & $50.4 \pm 20.1$ & $52.1 \pm 22.9$ & $44.6 \pm 15.1$ & $45.4 \pm 13.4$ & $71.4 \pm 22.1$ & $73.6 \pm 32.9$ \\
\hline DOC POST & $52.8 \pm 28.3$ & $51.5 \pm 22.0$ & $44.7 \pm 14.9$ & $46.3 \pm 15.9$ & $82.0 \pm 43.5$ & $68.2 \pm 30.1$ \\
\hline
\end{tabular}

Abbreviations: GTT gastrointestinal transit time, DOC duration of colour, STT short gastrointestinal transit time, $L T$ long gastrointestinal transit time, PRE pre-treatment, POST post-treatment.

${ }^{1}$ Mean \pm SD. 

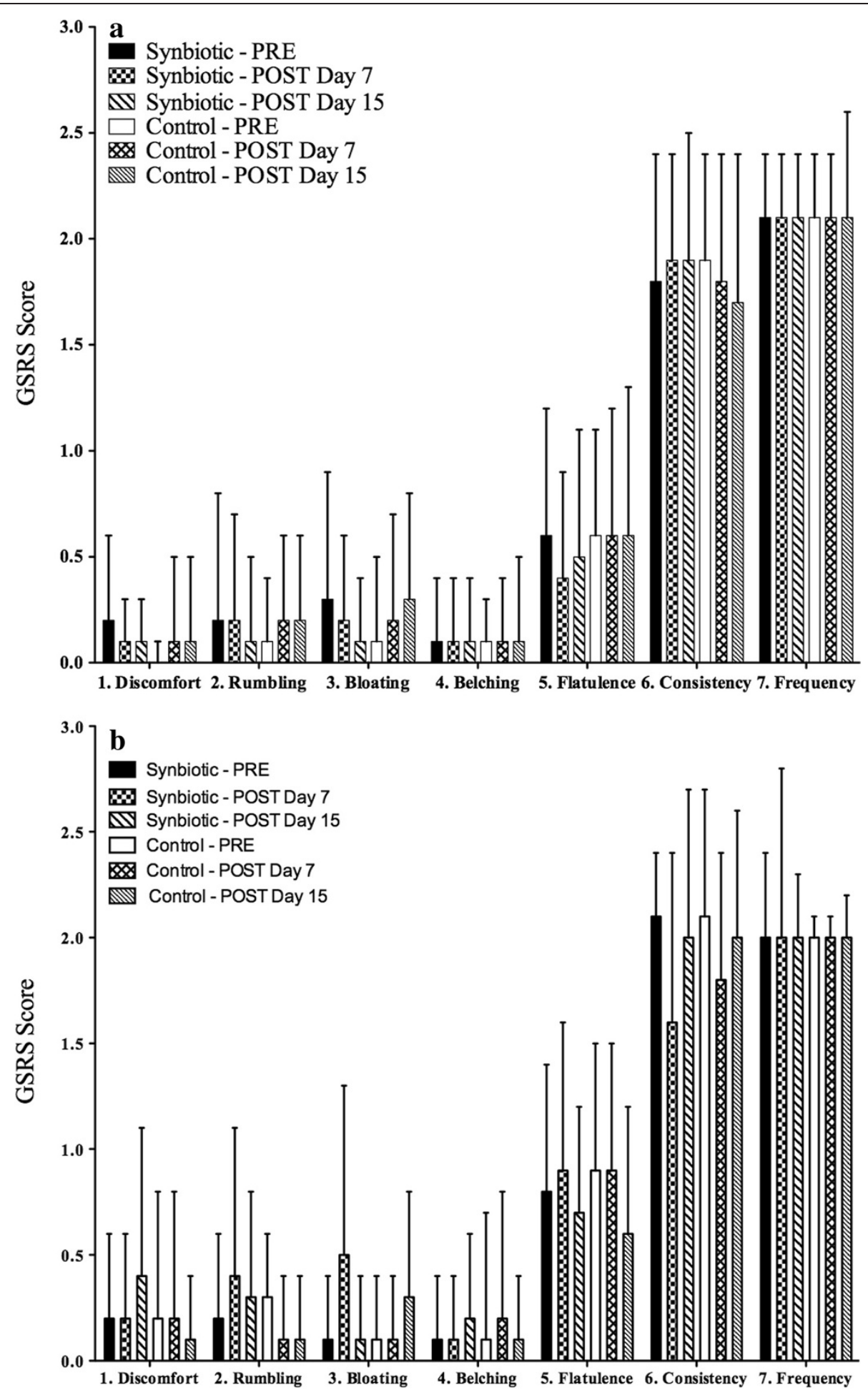

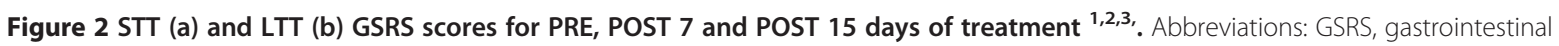
symptom rating scale; STT, short gastrointestinal transit time; LTT, long gastrointestinal transit time; PRE, pre-treatment; POST, post-treatment.

${ }^{1}$ Average GSRS scores. ${ }^{2}$ Mean \pm SD. ${ }^{3}$ Questions $1-5$ scored as 0 for 'no symptoms' to 3 for 'extreme symptoms' and questions 6 \& 7 scored as 0 to 4 , where 0 and 4 indicated opposite extremes and 2 indicated normal bowel movement consistency or frequency. 
Table 3 Average energy and macronutrient intakes at baseline and POST treatment ${ }^{1,2,3,4}$

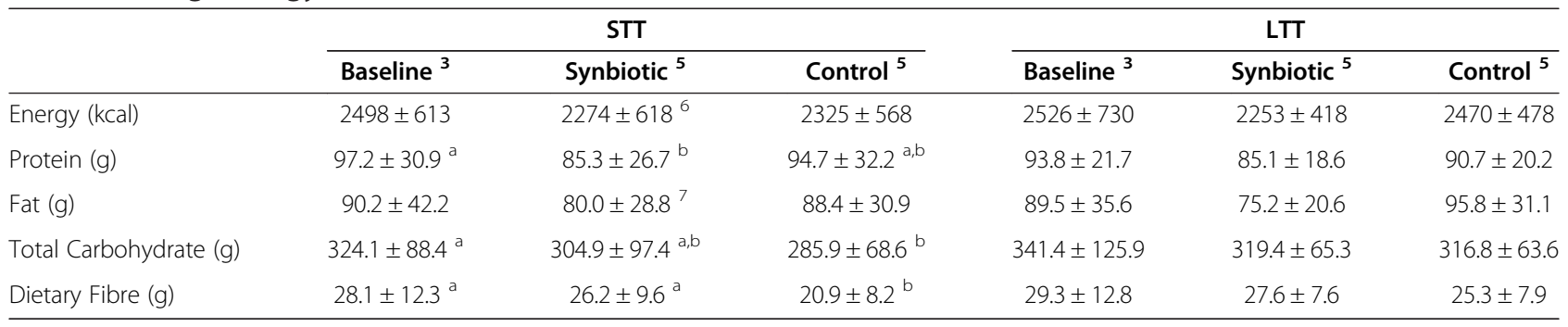

Abbreviations: STT short gastrointestinal transit time, $L T T$ long gastrointestinal transit time.

${ }^{1}$ Data are mean \pm SD.

${ }^{2}$ Means in a row with different superscript letters a \& b indicate a difference between treatments or from baseline within STT or LTT ( $<<0.05$ ).

${ }^{3}$ Based on 3-day food records completed at commencement of first washout period.

${ }^{4}$ Data includes contributions from the investigational yogurts.

${ }^{5}$ Based on 3-day food records completed on Days 13, 14 and 15 of each treatment period.

${ }^{6} \mathrm{p}=0.055$ between Baseline and Synbiotic.

$7 \mathrm{p}=0.059$ between Baseline and Synbiotic.

$\left(1 \times 10^{9}-6 \times 10^{9} \mathrm{CFU} / \mathrm{g}\right)$ daily for 15 days [20]. Similarly, consumption of 2 daily servings for 14 days of a commercial yogurt containing $B$. animalis $\mathrm{DN}-173$ $010\left(10^{8} \mathrm{CFU} / \mathrm{g}\right)$ and fructoligosaccharide by women with and without functional constipation improved measures of bowel evacuation [41]. Sairanen (2007) used radio-opaque marker detection in feces and, similar to the present study, found no improvements in transit time in healthy men and women whom, for 3 weeks, consumed $600 \mathrm{~mL}$ fermented milk with $12 \mathrm{~g}$ inulin and probiotics (Bifidobacterium longum BB536, Bifidobacterium spp. 420 and Lactobacillus acidophilus 145) versus fermented milk with the probiotics or a fermented milk control. In that study, average baseline transit times for the treatment groups ranged from $48.6 \pm 25.6$ to $53.5 \pm 22.4 \mathrm{~h}$.

There is consistent evidence to support the benefits of probiotic-enriched dairy products in improving GTT, even with relatively short nutrition interventions, if GTT is initially slow $[9,10,12,13]$. However, in healthy individuals, there is minimal room for improvement in GTT, the measurements of which can vary from 20 to $30 \%$, even with strict dietary controls $[47,51,52]$. Therefore, the relatively short initial GTT of the healthy and relatively young ( $28.6 \pm 11.3$ years) participants in the present study may help to explain the finding of no improvements in GTT. Of note, the physiological significance of small changes in GTT potentially induced in healthy individuals has not been confirmed. Conceivably, it would be undesirable for a product to excessively shorten a GTT such that it had laxative effects [30].

Differences between the results of the current study and those previously reported may be related to differences in probiotic strain and dosage, prebiotic type and concentration, and food matrix. The use of different techniques to determine GI transit may also explain apparent inconsistencies between studies. In the present study, use of the dye marker technique, while relatively non-invasive, inexpensive and conducive to studies with a relatively large number of participants, relies on participant self-reports of timing for dye ingestion and fecal appearance. It also cannot reveal transit times within specific segments of the GItract, where differences may exist [53].

Probiotic-containing foods are generally well tolerated, with minimal adverse side effects or undesirable GI symptoms [10]. However, the tolerability of products containing both probiotics and prebiotics is understudied and expected to be product-specific. In the current study, supplementing the daily diet with probiotic- and inulinenriched yogurt was well tolerated by healthy adults. Consumption of the same probiotic strains in yogurt also did not result in incidence of adverse GI symptoms beyond that observed with the placebo yogurt [21]. Similarly consumption of $10^{8}$ to $10^{11} \mathrm{CFU}$ of Bifidobacterium animalis and Lactobacillus paracasei per day did not induce adverse effects and was generally well tolerated in healthy adults [19]. In comparison to a milk control, milk fermented with Bifidobacterium lactis DN173010 was found to improve overall GI symptoms in women who reported minor digestive symptoms at baseline, mainly due to improvements in gas-related symptoms [14]. In contrast, intestinal discomfort from gas production has been implicated as a side effect of prebiotic consumption [1]. One study found that healthy adults experienced a significant increase in GI symptoms when consuming probiotic fermented milk with $12 \mathrm{~g}$ of inulin per day (4 $\mathrm{g}$ per $200 \mathrm{ml}$ serving) compared to the probiotic and control fermented milks [30]. However, inulin consumed in doses of 5 and $10 \mathrm{~g}$ is generally well tolerated [31]. Healthy adults whom consumed a supplement containing probiotics which included $\mathrm{Bb} 12$ and $\mathrm{La} 5$, as in the present study, reported beneficial effects on bowel habits [38]. A prebiotic dose of $5 \mathrm{~g} /$ day is reportedly sufficient to elicit a positive effect on the GI microbial population 
[1]. The current study delivered $4 \mathrm{~g}$ of inulin per day $(2 \mathrm{~g}$ per $100 \mathrm{~g}$ serving) and was well tolerated by healthy adults. Collectively, these results suggest that consumption of fermented dairy products, including or in conjunction with, a moderate amount of inulin $(\leq 10 \mathrm{~g}$ per day) does not induce undesirable GI symptoms in healthy adults $[34,54]$.

Analysis of dietary intake data revealed that energy intake was decreased in the STT and trended towards a decrease in the LTT during consumption of the synbiotic yogurt. The consumption of prebiotic fibres, including inulin-type fructans, can impact appetite-related endpoints and may play a role in appetite regulation and energy intake $[55,56]$. Reductions in daily energy intake by $100 \mathrm{kcal}$, similar to the $120 \mathrm{kcal}$ observed in this study can impact energy balance sufficiently to prevent weight gain [57]. This indicates that regular consumption of the synbiotic could translate into measurable and beneficial changes in dietary intake in free-living, healthy adults. In the LTT group, no differences in dietary fiber intake were observed between baseline and POST-treatment. However, in the STT group dietary fibre intake significantly decreased in the control group, but was maintained with consumption of the synbiotic yogurt. This indicates that consuming two daily servings of a synbiotic dairy product with $2 \mathrm{~g}$ inulin may reduce energy intake, while contributing dietary fibre. Since the average dietary fibre intake of adults in Canada and the United States fails to meet Adequate Intakes of $38 \mathrm{~g} /$ day for males and $25 \mathrm{~g} /$ day for females [58], foodbased strategies that support this aim are warranted. The current study is unique in that dietary intake was measured in healthy Canadian adults after 15 days of supplementing their diets with a synbiotic yogurt, whereas other studies have measured short-term hunger and satiety in laboratory settings [43-45]. Limitations of the study include the reliance on self-reported nutrient intake by participants and the fact that the significant reduction in energy intake was observed in the STT, but not the LTT group.

\section{Conclusion}

Consumption of a synbiotic yogurt containing Bifidobacterium lactis Bb12, Lactobacillus acidophilus La5, Lactobacillus casei CRL431 and inulin for 15 days did not significantly alter measures of GTT in a sample of 65 healthy Canadian adults. However, it was well tolerated according to GI symptom scores and its consumption was associated with reductions in energy intake. The study adds to the limited literature investigating the potential of synbiotic products to modulate GI function in healthy adults.

\section{Abbreviations}

Gl: Gastrointestinal; GTT: Gastrointestinal transit time; DOC: Duration of colour; STT: Short transit time; LTT: Long transit time; HNRU: Human Nutraceutical Research Unit.

\section{Competing interests}

The authors were solely responsible for study design, data collection, analysis, interpretation, manuscript preparation, and decision to publish the results.

\section{Authors' contributions}

HMFT \& DCB jointly contributed to preparation of the manuscript, data collection and analysis. LAM contributed to data collection and entry. AJW and $\mathrm{AMD}$ jointly led the study as co-principal investigators and supervised all personnel. All authors read and approved the final manuscript.

\section{Acknowledgements}

We gratefully acknowledge the study participants for their commitment to the study and thank Parmalat Dairy \& Bakery Inc. for their continued support. We thank Barbara Bowes, Edita Dolabchian, Dawn Robinson, and the HNRU undergraduate student volunteers for their help with data collection and Dr. William Bettger for his advice throughout the research process.

Received: 7 November 2012 Accepted: 22 May 2013

Published: 20 June 2013

\section{References}

1. Saulnier DM, Spinler JK, Gibson GR, Versalovic J: Mechanisms of probiosis and prebiosis: considerations for enhanced functional foods. Curr Opin Biotechnol 2009, 20:135-141.

2. Douglas LC, Sanders ME: Probiotics and prebiotics in dietetics practice. J Am Diet Assoc 2008, 108:510-521.

3. Ouwehand AC, Salminen S, Isolauri E: Probiotics: an overview of beneficial effects. Antonie Van Leeuwenhoek 2002, 82:279-289.

4. Sanders ME, Tompkins T, Heimbach JT, Kolida S: Weight of evidence needed to substantiatea health effect for probiotics and prebiotics. Eur J Nutr 2005, 44:303-310.

5. Heller KJ: Probiotic bacteria in fermented foods: product characteristics and starter organisms. Am J Clin Nutr 2001, 73:374S-379S.

6. Chmielewska A, Szajewska H: Systematic review of randomised controlled trials: probiotics for functional constipation. World J Gastroenterol 2010, 16:69-75.

7. de Vrese M, Schrezenmeir J: Probiotics, prebiotics, and synbiotics. Adv Biochem Eng Biotechnol 2008, 111:1-66.

8. Rodríguez-Fragoso L, Sandoval-Ocampo A, Corbalá-Nava M, Arjona-Canul CA, Gomez-Galicia DL, Tellez G, Hargis B, Reyes-Esparza J: Evaluation regarding the efficacy and safety of a probiotic mixture in healthy volunteers with evacuation disorders. Food Nutr Sci 2012, 3:117-122.

9. Yang $Y X$, He M, Hu G, Wei J, Pages P, Yang XH, Bourdu-Naturel S: Effect of a fermented milk containing Bifidobacterium lactis DN-173010 on Chinese constipated women. World J Gastroenterol 2008, 14:6237-6243.

10. Marteau P, Cuillerier E, Meance S, Gerhardt MF, Myara A, Bouvier M, Bouley C, Tondu F, Bommelaer G, Grimaud JC: Bifidobacterium animalis strain DN-173 010 shortens the colonic transit time in healthy women: a double-blind, randomized, controlled study. Aliment Pharmacol Ther 2002, 16:587-593.

11. Bouvier M, Meance S, Bouley C, Berta JL, Grimaud JC: Effects of consumption of a milk fermented by the probiotic strain Bifidobacterium animalis DN-173 010 on colonic transit times in healthy humans. Biosci Microflora 2001, 20(2):43-48.

12. Meance S, Cayuela C, Raimondi A, Turchet P, Lucas C, Antoine J: Recent advances in the Use of functional foods: effects of the commercial fermented milk with bifidobacterium animalis strain DN-173 010 and yoghurt strains on gut transit time in the elderly. Microb Ecol Health Dis 2003, 15:15-22.

13. Meance S, Cayuela C, Turchet P, Raimondi A, Lucas C, Antoine J-M: A fermented milk with a bifidobacterium probiotic strain DN-173 010 shortened Oro-fecal Gut transit time in elderly. Microb Ecol Health Dis 2001, 13:217-222.

14. Guyonnet D, Schlumberger A, Mhamdi L, Jakob S, Chassany O: Fermented milk containing Bifidobacterium lactis DN-173 010 improves gastrointestinal well-being and digestive symptoms in women reporting minor digestive symptoms: a randomised, double-blind, parallel, controlled study. Br J Nutr 2009, 102:1654-1662.

15. Nishida S, Ishikawa Y, lino H: Effect of Bifidobacterium lactis DN-173010 on the intestinal transit time, the condition of defecation and intestinal microflora: a randomized, double-blind, placebo-controlled, cross-over study among healthy Japanese women. Pharmacometrics 2008, 74(5/6):99-106. 
16. Ishizuka A, Tomizuka K, Aoki R, Nishijima T, Saito $Y$, Inoue R, Ushida K, Mawatari T, Ikeda T: Effects of administration of Bifidobacterium animalis subsp. lactis GCL2505 on defecation frequency and bifidobacterial microbiota composition in humans. J Biosci Bioeng 2012, 113:587-591.

17. Saejong R: Effect of fermented milk containing probiotics on constipation symptoms and immune system in subjects with constipation. Siriraj Med J 2012, 64:105-109.

18. Yamano $T$, lino $H$, Takada $M$, Blum $S$, Rochat $F$, Fukushima $Y$ : Improvement of the human intestinal flora by ingestion of the probiotic strain Lactobacillus johnsonii La1. Br J Nutr 2006, 95:303-312.

19. Larsen CN, Nielsen S, Kæstel P, Brockmann E, Bennedsen M, Christensen HR, Eskesen DC, Jacobsen BL, Michaelsen KF: Dose-response study of probiotic bacteria Bifidobacterium animalis subsp lactis BB-12 and Lactobacillus paracasei subsp paracasei CRL-341 in healthy young adults. Eur J Clin Nutr 2006, 60:1284-1293.

20. Malpeli A, González S, Vicentin D, Apás A, González HF: Randomised, double-blind and placebo-controlled study of the effect of a synbiotic dairy product on orocecal transit time in healthy adult women. Nutrición hospitalaria : organo oficial de la Sociedad Española de Nutrición Parenteral y Enteral 2012, 27:1314-1319.

21. Savard P, Lamarche B, Paradis M, Thiboutot H, Laurin E, Roy D: Impact of Bifidobacterium animalis subsp. lactis BB-12 and, Lactobacillus acidophilus LA-5-containing yoghurt, on fecal bacterial counts of healthy adults. Int J Food Microbiol 2011, 149:50-57.

22. Wang KY, Li SN, Liu CS, Perng DS, Su YC, Wu DC, Jan CM, Lai CH, Wang TN, Wang WM: Effects of ingesting Lactobacillus- and Bifidobacteriumcontaining yogurt in subjects with colonized Helicobacter pylori. Am J Clin Nutr 2004, 80:737-741.

23. Ejtahed HS, Mohtadi-Nia J, Homayouni-Rad A, Niafar M, Asghari-Jafarabadi M, Mofid V: Probiotic yogurt improves antioxidant status in type 2 diabetic patients. Nutrition 2012, 28:539-543.

24. Asemi Z, Samimi M, Tabassi Z, Naghibi Rad M, Rahimi Foroushani A, Khorammian $\mathrm{H}$, Esmaillzadeh A: Effect of daily consumption of probiotic yoghurt on insulin resistance in pregnant women: a randomized controlled trial. Eur J Clin Nutr 2013, 67:71-74.

25. Gibson GRG, Roberfroid MBM: Dietary modulation of the human colonic microbiota: introducing the concept of prebiotics. J Nutr 1995, 125:1401-1412.

26. Meyer $D$, Stasse-Wolthuis M: The bifidogenic effect of inulin and oligofructose and its consequences for gut health. Eur J Clin Nutr 2009, 63:1277-1289.

27. Den Hond E, Geypens B, Ghoos Y: Effect of high performance chicory inulin on constipation. Nutr Res 2000, 20:731-736.

28. Kleessen BB, Sykura BB, Zunft HJH, Blaut MM: Effects of inulin and lactose on fecal microflora, microbial activity, and bowel habit in elderly constipated persons. Am J Clin Nutr 1997, 65:1397-1402.

29. Cani PD, Lecourt E, Dewulf EM, Sohet FM, Pachikian BD, Naslain D, De Backer F, Neyrinck AM, Delzenne NM: Gut microbiota fermentation of prebiotics increases satietogenic and incretin gut peptide production with consequences for appetite sensation and glucose response after a meal. Am J Clin Nutr 2009, 90:1236-1243.

30. Sairanen U, Piirainen L, Grasten S, Tompuri T, Maettoe J, Saarela M, Korpela R: The effect of probiotic fermented milk and inulin on the functions and microecology of the intestine. J Dairy Res 2007, 74:367-373.

31. Bonnema ALA, Kolberg LWL, Thomas WW, Slavin JLJ: Gastrointestinal tolerance of chicory inulin products. J Am Diet Assoc 2010, 110:865-868.

32. Roberfroid MB: Prebiotics and probiotics: are they functional foods? Am J Clin Nutr 2000, 71:1682S-1687S. discussion 1688S-90S

33. Leovaridis C: Comparative aspects regarding consumption and consumers in European countries. Manage Mark 2010, 8:247-257.

34. Lourens-Hattingh A, Viljoen BC: Yogurt as probiotic carrier food. Int Dairy J 2001, 11:1-17

35. Weenen $\mathrm{H}$, Jellema $\mathrm{RH}$, de Wilk R: Prediction of creamy mouthfeel based on texture attribute ratings of dairy desserts. Food Lipid 2005, 920:105-117.

36. Desai AR, Powell IB, Shah NP: Survival and activity of probiotic lactobacill in skim milk containing prebiotics. J Food Sci 2004, 69:FMS57-FMS60.

37. Capela P, Hay TKC, Shah NP: Effect of cryoprotectants, prebiotics and microencapsulation on survival of probiotic organisms in yoghurt and freeze-dried yoghurt. Food Res Int 2006, 39:203-211.

38. Nova EE, Viadel BB, Wärnberg JJ, Carreres JEJ, Marcos AA: Beneficial effects of a synbiotic supplement on self-perceived gastrointestinal well-being and immunoinflammatory status of healthy adults. J Med Food 2011 14:79-85.

39. Fateh R, Iravani S, Frootan M, Rasouli MR, Saadat S: Synbiotic preparation in men suffering from functional constipation: a randomised controlled trial. Swiss Med Wkly 2011, 141:w13239.

40. Khodadad A, Sabbaghian M: Role of synbiotics in the treatment of childhood constipation: a double-blind randomized placebo controlled trial. Iran J Pediatr 2010, 20:387-392.

41. De Paula JA, Carmuega E, Weill R: Effect of the ingestion of a symbiotic yogurt on the bowel habits of women with functional constipation. Acta Gastroenterol Latinoam 2008, 38:16-25.

42. Waitzberg DL, Logullo LC, Bittencourt AF, Torrinhas RS, Shiroma GM, Paulino NP, Teixeira-da-Silva ML: Effect of synbiotic in constipated adult women - a randomized, double-blind, placebo-controlled study of clinical response. Clin Nutr (Edinburgh, Scotland) 2013, 32:27-33.

43. Lluch A, Hanet-Geisen N, Salah S, Salas-Salvado J, L'Heureux-Bouron D, Halford JCG: Short-term appetite-reducing effects of a low-fat dairy product enriched with protein and fibre. Food Qual Preference 2010, 21:402-409.

44. Perrigue MM, Monsivais $P$, Drewnowski A: Added soluble fiber enhances the satiating power of low-energy-density liquid yogurts. J Am Diet Assoc 2009, 109:1862-1868.

45. Tsuchiya A, Almiron-Roig E, Lluch A, Guyonnet D, Drewnowski A: Higher satiety ratings following yogurt consumption relative to fruit drink or dairy fruit drink. J Am Diet Assoc 2006, 106:550-557.

46. Tsuchiya A, Almiron-Rong E, Lluch A, Guyonnet D, Drewnowski A: Added soluble fiber enhances the satiating power of low energy density lquid yogurts. J Am Diet Assoc 2009, 109:1862-1868.

47. Cummings JHJ, Antoine JJ, Azpiroz FF, Bourdet-Sicard RR, Brandtzaeg PP, Calder PCP, Gibson GRG, Guarner FF, Isolauri EE, Pannemans DD, Shortt CC, Tuijtelaars SS, Watzl BB: PASSCLAIM-gut health and immunity. Eur J Nutr 2004, 43(Suppl 2):|1118-||173.

48. Rose GAG: Experiences with the use of interrupted carmine red and continuous chromium sesquioxide marking of human faeces with reference to calcium, phosphorus, and magnesium. Gut 1964, 5:274-279.

49. Walker ARA, Walker BFB: Bowel motility and colonic cancer. Br Med J 1969, $3: 238$

50. Svedlund JJ, Sjödin II, Dotevall GG: GSRS-a clinical rating scale for gastrointestinal symptoms in patients with irritable bowel syndrome and peptic ulcer disease. Dig Dis Sci 1988, 33:129-134.

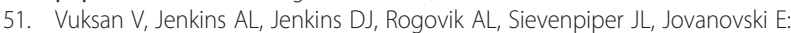
Using cereal to increase dietary fiber intake to the recommended level and the effect of fiber on bowel function in healthy persons consuming North American diets. Am J Clin Nutr 2008, 88:1256-1262.

52. Wyman JB, Heaton KW, Manning AP, Wicks AC: Variability of colonic function in healthy subjects. Gut 1978, 19:146-150.

53. Ibrahim F, Ouwehand AC: Potential of probiotics in reducing intestinal transit time. Touch Brief 2011:1-4.

54. Kolida SS, Gibson GRG: Prebiotic capacity of inulin-type fructans. J Nutr 2007, 137:2503S-2506S.

55. Delzenne NM, Cani PD, Neyrinck AM: Modulation of glucagon-like peptide 1 and energy metabolism by inulin and oligofructose: experimental data. Journal of nutrition 2007, 137:2547S-2551S

56. Delzenne NM, Neyrinck AM, Cani PD: Gut microbiota and metabolic disorders: How prebiotic can work? Br J Nutr 2013, 109(Suppl 2):S81-S85.

57. Hill JO, Wyatt HR, Reed GW, Peters JC: Obesity and the environment: Where do we go from here? Science 2003, 299:853-855.

58. Trumbo P, Schlicker $S$, Yates AA, Poos M: Dietary reference intakes for energy, carbohydrates, fiber, fat, fatty acids, cholesterol, protein, and amino acids. J Am Diet Assoc 2002, 102:1621-1630.

doi:10.1186/1475-2891-12-87

Cite this article as: Tulk et al:: Daily consumption of a synbiotic yogurt decreases energy intake but does not improve gastrointestinal transit time: a double-blind, randomized, crossover study in healthy adults. Nutrition Journal 2013 12:87. 